\title{
Escarabajos estercoleros (Coleoptera: Scarabaeidae: Scarabaeinae) de ranchos ganaderos de Yucatán, México
}

\section{Dung beetles (Coleoptera: Scarabaeidae: Scarabaeinae) from cattle ranches of Yucatán, Mexico}

\author{
Gertrudis Basto-Estrella ${ }^{1}$, Roger Iván Rodríguez-Vivas ${ }^{1}$, Hugo Delfín-González y Enrique Reyes-Novelo ${ }^{2 \otimes}$ \\ ${ }^{1}$ Universidad Autónoma de Yucatán, Campus de Ciencias Biológicas y Agropecuarias. Carretera Mérida-Xmatkuil s/n Km. 15.5, 97000 Mérida, Yuca- \\ tán, México. \\ ${ }^{2}$ Centro de Investigaciones Regionales Dr. Hideyo Noguchi, Universidad Autónoma de Yucatán, Av. Itzaes $490 \times 59$, Col. Centro, 97000 Mérida, \\ Yucatán, México. \\ 凶enrique.reyes@uady.mx
}

\begin{abstract}
Resumen. Este estudio describe la fauna de escarabajos estercoleros (Scarabaeidae: Scarabaeinae) en ranchos ganaderos del trópico subhúmedo. De enero a diciembre de 2010 se recolectaron 93274 escarabajos estercoleros pertenecientes a 17 especies de la subfamilia Scarabaeinae con trampas de caída libre cebadas con excremento de ganado bovino en 4 ranchos ganaderos del oriente del estado de Yucatán, México. Las especies más abundantes fueron Onthophagus landolti, seguida por Canthon indigaceus chevrolati, Digitonthophagus gazella, C. leechi y Pseudocanthon perplexus. Se observó que las especies cavadoras tuvieron mayor abundancia y riqueza; las diurnas de talla pequeña tuvieron menor riqueza pero fueron más abundantes en todo el estudio. El conocimiento de la composición faunística de escarabajos estercoleros de la región es un punto de partida para desarrollar estudios del impacto de las actividades humanas y las prácticas de producción ganadera sobre la diversidad de estos insectos.
\end{abstract}

Palabras clave: riqueza, especies, diversidad, ganadería, estiércol, escarabajos.

\begin{abstract}
This study describes the fauna of dung beetles (Scarabaeidae: Scarabaeinae) in tropical sub-humid cattle ranches. Insects were collected with pitfall traps baited with cattle manure from January to December 2010 in four cattle ranches of eastern state of Yucatán, Mexico. 93274 dung beetles were collected from 17 species of the subfamily Scarabaeinae. The most abundant species were Onthophagus landolti, followed by Canthon indigaceus chevrolati, Digitonthophagus gazella, C. leechi y Pseudocanthon perplexus. Observations showed that burrowing species had greater abundance and species richness. Diurnal species of small size had lower richness, but were more abundant. Knowledge of the composition of dung beetle fauna of the region is a starting point to develop studies of the impact of human activities and livestock production practices on the diversity of these insects.
\end{abstract}

Key words: richness, species, diversity, cattle rising, dung, beetles.

\section{Introducción}

Los escarabajos estercoleros de la subfamilia Scarabaeinae son un grupo clave para la sustentabilidad de los pastizales (Steinfeld et al., 2006), ya que participan activamente en el reciclaje de nutrientes en el suelo, incorporando la materia orgánica en descomposición o de desecho producida por animales vertebrados y que estos insectos utilizan como alimento tanto para adultos como para estados inmaduros (Halffter y Edmonds, 1982). Con ésto, reducen la pérdida de elementos nitrogenados en las áreas de producción de forraje (potreros) y contribuyen a incrementar la fertilidad del suelo al acelerar la incorporación del estiércol al ciclo de nutrientes. Adicionalmente,

Recibido: 29 julio 2011; octubre 2011 intervienen como agentes de control biológico de nematodos gastrointestinales y de larvas de algunos dípteros perjudiciales para el ganado que cumplen su ciclo de vida en las excretas (Bishop et al., 2005; Nichols et al., 2008). Los Scarabaeinae presentan amplia distribución geográfica y pueden llegar a colonizar una gran variedad de hábitats, son abundantes y diversos en regiones tropicales; dicha diversidad decrece en regiones con bajas temperaturas hasta estar ausentes en regiones frías (Halffter y Edmonds, 1982; Halffter, 1991; Hanski y Cambefort, 1991).

Se conocen alrededor de 6000 especies y 200 géneros de escarabajos estercoleros en el mundo, concentrándose una buena parte en la zona tropical con cerca de 1300 especies y alrededor de 70 géneros (Halffter y Edmonds, 1982; Halffter, 1991; Morón, 2003). En México existen 228 especies de Scarabaeinae distribuidas en 25 géneros (Morón, 2003). Para Yucatán, diversos trabajos han regis- 
trado 28 especies pertenecientes a 16 géneros (Edmonds, 2003; Halffter, 2003; Kohlmann, 2003; Zunino, 2003; Deloya y Peraza, 2005; Delgado et al., 2006; Kohlmann y Solís, 2006; Peraza y Deloya, 2006; Delgado y Kohlmann, 2007; Reyes-Novelo et al., 2007). La mayoría de estas especies se encuentran ampliamente distribuidas en el país y en América Central; sin embargo, algunas de las descripciones recientes tienen su distribución restringida a la provincia de la península de Yucatán, como Dichotomius maya, Onthophagus yucatanus, Uroxys chichanich y U. deavilai (Delgado et al., 2006; Peraza y Deloya, 2006; Delgado y Kohlmann, 2007).

En el único estudio publicado en ambientes ganaderos de Yucatán, los autores registraron 11 especies en el potrero de un rancho ubicado en la parte norte de la región (Reyes-Novelo et al., 2007). En dicho estudio predominaron las especies de talla pequeña, hubo mayor riqueza de especies nocturnas y mayor abundancia de diurnas. En otros estudios realizados en Veracruz se ha registrado un número similar de especies: Estrada et al. (1998) y Halffter y Arellano (2002) registraron 9 de Scarabaeinae y Montes de Oca (2001) 11. Adicionalmente, en un estudio realizado en un pastizal en El Salvador se encontraron 15 especies, siendo las más abundantes las cavadoras diurnas de talla grande (Horgan, 2001). La aparente escasez en las áreas ganaderas facilita su estudio y permite conocer a profundidad la composición de sus comunidades. Por lo tanto, el objetivo de este trabajo es describir la fauna de escarabajos estercoleros Scarabaeinae que habita en potreros de la zona ganadera del oriente del estado de Yucatán.

\section{Materiales y métodos}

El estudio se realizó de enero a diciembre de 2010 en 4 ranchos bovinos localizados en la zona ganadera del oriente del estado de Yucatán, México (municipio de Tizimín), ubicada en los $07^{\circ} 58^{\prime} \mathrm{N}$ y $88^{\circ} 09^{\prime} \mathrm{O}$. El clima de la región es cálido subhúmedo con precipitación total anual de 1 000-1 $200 \mathrm{~mm}$ y temperatura promedio anual de 25.8 ${ }^{\circ} \mathrm{C}$ (Navarro-Reyes, 2005). La geomorfología pertenece al sistema carsotectónico joven, clasificado como un paisaje geomorfológico de planicie estructural ondulada con disolución y denudación. Los suelos son principalmente de tipo litosol, rendzina y cambisol (Bautista et al., 2005).

Actualmente, sólo se puede observar vegetación secundaria de selva baja y mediana caducifolia en diferentes estados de regeneración, localizada en parches entre los potreros de pastos Brachiaria brizantha (Hochst. ex A. Rich.) Stapf. y Panicum maximun (Jacq.) B. K. Simon y S. W. L. Jacobs. Dichos fragmentos están conformados por Caesalpinia gaumeri Greenm. (Fabaceae), Piscidia piscipula (L.) Sarg. (Fabaceae), Mimosa bahamensis
Benth. (Fabaceae), Havardia albicans (Kunth.) Benth. (Fabaceae), Acacia gaumeri Blake (Fabaceae), Randia longiloba Hamsley (Rubiaceae), Ceiba schottii Britton y Baker (Bombacaceae), Bursera simaruba (L.) Sarg. (Burceraceae), Helicteres baruensis Jacq. (Sterculiaceae), Diospyros cuneata Standley (Ebenaceae) y Thouinia paucidentata Radlk (Sapindaceae), entre las especies más comunes en la zona (Flores y Espejel, 1994). Los 4 ranchos estudiados se dedican a la producción de carne bovina y se localizan en los 4 puntos cardinales del municipio, Actun-Há al norte, Santa Rosa al sur, Pedregal al este y San Carlos al oeste.

Para recolectar los escarabajos estercoleros se colocaron en cada rancho 2 transectos separados por $500 \mathrm{~m}$ entre sí, cada transecto estuvo conformado por 3 coprotrampas de caída libre o pitfall cebadas con $200 \mathrm{~g}$ de excremento bovino y separadas $200 \mathrm{~m}$ entre sí para mantener la independencia de las muestras (Larsen y Forsyth, 2005). Las trampas estuvieron activas durante 48 horas, con recolección de ejemplares y reposición de cebo cada 24 horas. El muestreo fue mensual durante un año (2010) y los escarabajos recolectados se transportaron y almacenaron en frascos de plástico con alcohol al $70 \%$, para su posterior determinación.

La determinación genérica se realizó de acuerdo con los criterios de Morón (2003) y Vaz-de-Mello et al. (2011) y para la específica se usaron diferentes trabajos monográficos, claves y descripciones, como los de Rivera-Cervantes y Halffter (1999), Kohlmann y Solís (1997, 2006), Peraza y Deloya (2006) y Delgado y Kohlmann (2007). Adicionalmente, el material recolectado se comparó con una colección de referencia, determinada por M. A. Morón, la cual se generó durante el trabajo de Reyes-Novelo et al. (2007). Todos los ejemplares se encuentran depositados en la Colección Entomológica Regional de la Universidad Autónoma de Yucatán (CER-UADY). Se elaboraron curvas de acumulación de especies con los datos observados para cada sitio y se estimó la riqueza de especies con los modelos de ACE y Chao1, calculados con Estimates ver. 8.20 (Colwell, 2009).

\section{Resultados}

Se recolectaron 93274 ejemplares pertenecientes a 17 especies distribuidas en 10 géneros (Cuadro 1). La más abundante fue Onthophagus landolti con el $61 \%$ de los individuos, seguida por Canthon (C.) indigaceus chevrolati (30.3\%), Digitonthophagus gazella (2.8\%), C. (Glaphyrocanthon) leechi $(2.4 \%)$ y Pseudocanthon perplexus $(2.1 \%)$, el restante $1.4 \%$ estuvo distribuido entre las otras 12 especies. Para 14 de las 17 especies ya se tenían registros de colecta en Yucatán, las otras 3 representan los 
Cuadro 1. Scarabaeinae recolectados en Tizimín, Yucatán, México

\begin{tabular}{|c|c|c|c|c|}
\hline Especies & Abundancia & Espacial & Temporal & Tamaño \\
\hline \multicolumn{5}{|l|}{ Coprini } \\
\hline \multicolumn{5}{|l|}{ Coprina } \\
\hline Copris laeviceps Harold, 1869* & 134 & cavador & nocturno & mediano \\
\hline C. lugubris Boheman, 1858 & 236 & cavador & nocturno & grande \\
\hline \multicolumn{5}{|l|}{ Dichotomina } \\
\hline Canthidium pseudopuncticolle Solís y Kohlmann, 2004 & 223 & cavador & nocturno & pequeño \\
\hline Dichotomius maya Peraza y Deloya, 2006 & 57 & cavador & nocturno & grande \\
\hline D. yucatanus Bates, 1887 & 1 & cavador & nocturno & grande \\
\hline Uroxys chichanich Delgado y Kohlmann, 2007 & 32 & cavador & nocturno & pequeño \\
\hline \multicolumn{5}{|l|}{ Eurysternini } \\
\hline $\begin{array}{l}\text { Eurysternus mexicanus Harold, } 1869^{*} \\
\text { Phanaeini }\end{array}$ & 52 & cavador & nocturno & mediano \\
\hline \multicolumn{4}{|l|}{ Phanaeina } & grande \\
\hline \multicolumn{5}{|l|}{ Onthophagini } \\
\hline Onthophagus landolti Harold, 1880 & 56982 & cavador & diurno & pequeño \\
\hline $\begin{array}{l}\text { Digitonthophagus gazella Fabricius, 1787* } \\
\text { Scarabaeini }\end{array}$ & 2608 & cavador & diurno & mediano \\
\hline \\
\hline C. (Glaphyrocanthon) leechi Martínez, Halffter y Halffter, 1964 & 2240 & rodador & diurno & pequeño \\
\hline Deltochilum (Hybomidium) lobipes Bates, 1887 & 4 & rodador & nocturno & grande \\
\hline Deltochilum (D.) scabriusculum scabriusculum Bates, 1887 & 15 & rodador & nocturno & grande \\
\hline Malagoniella (M.) astyanax yucateca Harold, 1863 & 11 & rodador & nocturno & grande \\
\hline Pseudocanthon perplexus LeConte, 1847 & 1989 & rodador & nocturno & pequeño \\
\hline \multicolumn{5}{|l|}{ Sisyphina } \\
\hline Sisyphus mexicanus Harold, 1863 & 333 & rodador & diurno & pequeño \\
\hline
\end{tabular}

* Primer registro para el estado de Yucatán.

primeros registros para el estado (Cuadro 1). De acuerdo con las curvas de acumulación de especies (Fig. 1) y los estimadores de riqueza (Cuadro 2), el inventario de Scarabaeinae coprófagas que habitan en los potreros de la región se puede considerar representativo para estos ambientes. Ambas valoraciones indican que existen pocas posibilidades de encontrar más especies en los potreros.

La segregación espacial mostró que de las 17 especies 7 fueron rodadoras y 10 cavadoras, siendo estas últimas las que presentaron las mayores abundancias (Fig. 2). En la segregación temporal se encontró que 6 especies fueron diurnas y 11 nocturnas; las mayores abundancias las presentaron las diurnas, una cavadora $(O$. landolti) y una rodadora ( $C$. indigaceus chevrolati). Se puede observar claramente que se encontraron escarabajos a lo largo de todo el año; sin embargo, las mayores abundancias fluctuaron de abril a septiembre y en diciembre se recolectó la menor riqueza (Fig. 2).

\section{Discusión}

La fauna de Scarabaeinae de Yucatán ha sido poco estudiada, ya que en los últimos diez años sólo se desarro- llaron 3 trabajos formales, de los cuales se ha derivado un listado de 31 especies válidas. No obstante, dichos trabajos señalan que la riqueza de especies no es muy alta para esta familia, y considerando algunos registros informales y datos no publicados de recolecciones esporádicas, es posible que la fauna del estado tenga pocas especies por documentar. Comparando con la referencia más cercana para la región, en el presente estudio se encontró mayor riqueza de especies y 15 veces más ejemplares que lo presentado en el trabajo de Reyes-Novelo et al. (2007). Probablemente, la elevada cantidad de escarabajos recolectados en este estudio se debió a la disponibilidad de excremento de ganado vacuno, recurso que se encuentra de

Cuadro 2. Estimadores de la riqueza de Scarabaeinae en potreros de 4 ranchos de Tizimín, Yucatán, México

\begin{tabular}{lcccc}
\hline Ranchos & $\begin{array}{c}\text { Especies } \\
\text { observadas }\end{array}$ & ACE & Chao 1 & $\%$ \\
\hline Pedregal & 14 & 14.63 & 14 & $96-100$ \\
San Carlos & 15 & 15.60 & 15 & $96-100$ \\
Santa Rosa & 17 & 17.54 & 17 & $97-100$ \\
Actun-Há & 13 & 13.45 & 13 & $97-100$ \\
\hline
\end{tabular}




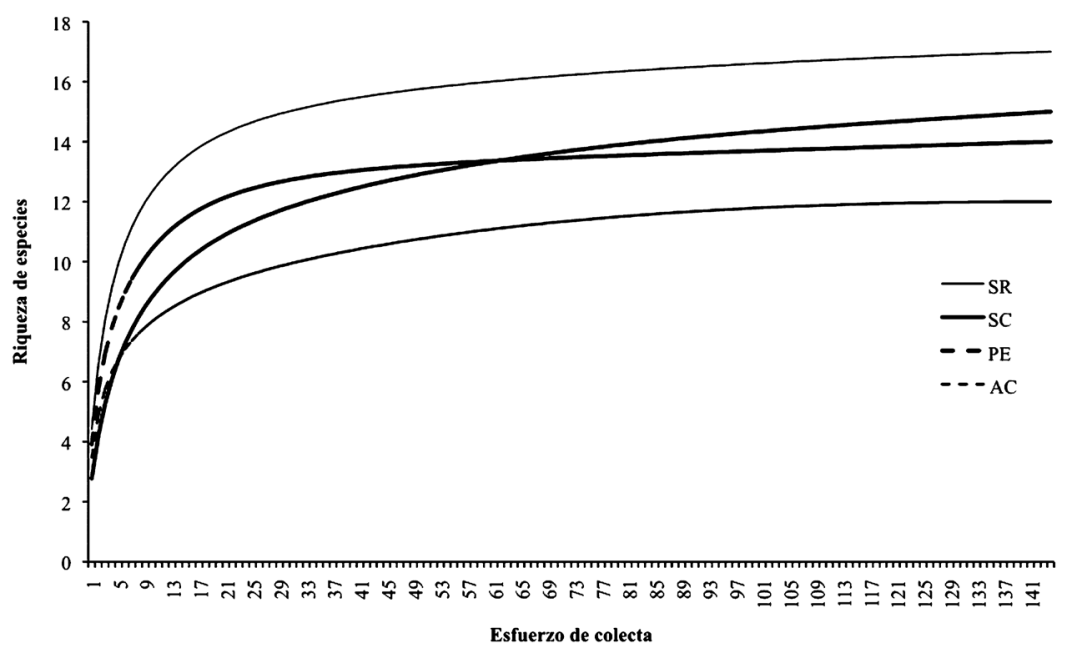

Figura 1. Curvas de acumulación de especies de Scarabaeinae colectados en potreros de 4 ranchos ganaderos de Tizimín, Yucatán. SR, Santa Rosa; SC, San Carlos; PE, Pedregal y AC, Actun-Há.

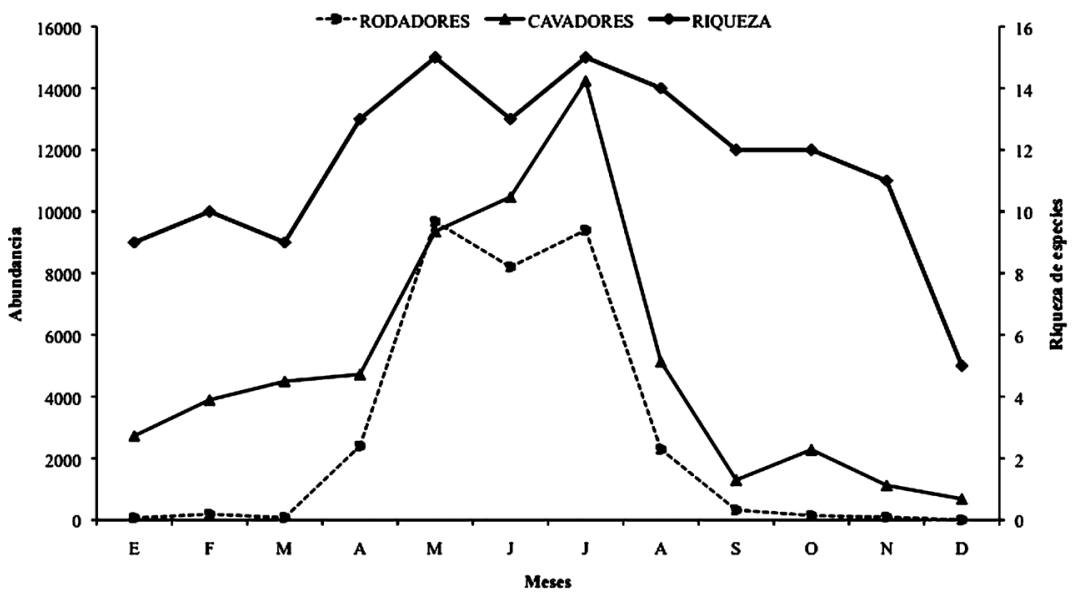

Figura 2. Fenología de la riqueza de especies durante el año 2010 y abundancia de escarabajos estercoleros rodadores y cavadores en 4 ranchos ganaderos de Tizimín, Yucatán.

forma abundante en la zona, ya que diferentes trabajos han documentado el uso extenso que algunas especies pueden hacer de este recurso, en ausencia o lejanía de excremento de animales silvestres y otras formas de materia orgánica en descomposición (Halffter, 1991; Hanski y Cambefort, 1991; García y Pardo, 2004).

De las 17 especies recolectadas en este estudio, 10 comparten lo encontrado por Reyes-Novelo et al. (2007) (C. indigaceus chevrolati, C. leechi, Copris lugubris, Canthidium psedopuncticolle, Deltochilum scabriusculum, D. lobipes, Malagoniella astyanax yucateca, O. landolti, P. perplexus y Uroxys chichanich). En ambos estudios se encuentra predominio de escarabajos pequeños y mayor riqueza de nocturnos, pero mayor abundancia de diurnos; sin embargo, es interesante notar que en este estudio hubo mayor abundancia y riqueza de cavadores, mientras que en el norte del estado se observó una mayor abundancia y riqueza de rodadores. Es probable que esta diferencia esté relacionada con las características del suelo, ya que Halffter (1991) menciona que el suelo es un factor fundamental para el entendimiento de la diversidad de Scarabaeinae, porque en él se desarrolla la mayor parte de su ciclo de vida. En este sentido, Reyes-Novelo et al. (2007) señalan que en el norte de Yucatán los suelos son poco profundos y muy rocosos, lo que favorece a los escarabajos rodadores que hacen nidos poco profundos. En contraste, en la región de Tizimín, las características de los suelos cambia, ya que son más profundos y menos pedregosos (Bautista et al., 2005), favoreciendo la presencia de escarabajos cavadores. 
En varios estudios se encontró que al transformar la vegetación original en áreas de potrero, el número de especies de estos escarabajos disminuye notoriamente (Howden y Nealis, 1975; Klein, 1989; Halffter, 1991; Hansky y Cambenfort, 1991; Montes de Oca y Halffter, 1995; Horgan, 2001; Halffter y Arellano, 2002), lo que puede deberse a la alta exposición al viento y a la radiación solar que dan como resultado condiciones de temperatura y humedad no adecuadas para muchas especies (Hanski, 1989; Halffter, 1991; Hanski y Cambefort, 1991; Gill, 1991), aunque también es probable que la menor complejidad estructural de la vegetación afecte la riqueza de estos escarabajos (Estrada et al., 1998) y genere mayor dominancia de algunas de las especies asociadas con ambientes abiertos y donde hay gran disponibilidad de excremento (Halffter et al., 1992; Montes de Oca, 2001).

Las 2 especies que presentaron mayor abundancia $(O$. landolti y $C$. indigaceus chevrolati) parecen tener una tolerancia ecológica amplia, ya que $O$. landolti es una especie abundante en áreas tropicales y subtropicales por debajo de los $1500 \mathrm{~m} \mathrm{snm}$, explotando sobre todo las heces de mamíferos herbívoros, mientras que $C$. indigaceus chevrolati se encuentra en selvas siempre verdes, claros, desmontes, bosque tropical, deciduo y espinoso, desde el nivel del mar hasta los $1800 \mathrm{~m}$ (Morón, 2003). Reyes-Novelo et al. (2007) registraron 4 especies dominantes en potrero, entre ellas $O$. landolti y $C$. indigaceus chevrolati. Estas especies coexisten en áreas con presencia de grandes cantidades de estiércol (Hanski y Cambefort, 1991; Montes de Oca, 2001); sin embargo, las abundancias observadas en este estudio rebasan por mucho las descritas por Reyes-Novelo et al. (2007), y minimizan la abundancia de otras especies, como C. leechi y C. pseudopuncticole, las cuales fueron especies dominantes en el citado estudio.

Estudios faunísticos y ecológicos efectuados en diversas zonas tropicales han mostrado la relación que existe entre la época de lluvia y la actividad y ciclo de vida de los escarabajos estercoleros (Halffter y Edmonds, 1982; Janzen 1983; Wolda, 1988; Martínez y Montes de Oca, 1994; Barraza et al., 2010). Durante este periodo se pueden observar marcados cambios en la riqueza y abundancia de las comunidades de coprófagos, las cuales presentan sus picos máximos, mientras que durante el periodo seco muestran un señalado descenso. Se sabe que la actividad de los escarabajos estercoleros se ve limitada durante las épocas secas debido a que no pueden elaborar galerías en el suelo por la dureza y compactación del mismo; por tanto, los insectos permanecen enterrados y no salen a la superficie (Halffter y Edmonds, 1982; Janzen, 1983; Breytenbanch y Breytenbanch, 1986; Rougon y Rougon, 1991; Martínez y Montes de Oca, 1994; Escobar, 1997; Halffter y Arellano, 2002; Andresen, 2005; Barraza et al., 2010). En este estudio se observó el mismo patrón fenológico (Hanski y Cambefort, 1991; Martínez y Montes de Oca, 1994) ya que la mayor abundancia se obtuvo durante los meses de mayo a agosto, los cuales corresponden al periodo de lluvias en la región.

En términos generales, la comunidad de escarabajos estercoleros Scarabaeinae que habita en potreros de la zona ganadera del oriente del estado de Yucatán está integrada por 17 especies, con predominio de especies cavadoras, tanto en riqueza como en abundancia. Aun cuando la riqueza de especies diurnas y de talla pequeña fue menor, fueron las más abundantes, principalmente $O$. landolti. En este sentido, el conocimiento de la composición faunística de escarabajos estercoleros de las áreas ganaderas de la región es un punto de partida para la conformación de estudios específicos sobre el impacto de las actividades humanas y de las prácticas de producción ganadera sobre la diversidad de estos insectos, los cuales desempeñan un papel indispensable en el reciclaje de nutrientes en estos sistemas productivos y por tanto en la sustentabilidad de su funcionamiento.

\section{Agradecimientos}

Este proyecto fue financiado por CONACYT (Ciencia Básica 128870) y por la beca de posgrado de GSBE (97831). Se agradece a los ganaderos de Tizimín, Yucatán, quienes proporcionaron las facilidades para desarrollar este estudio en sus ranchos. Virginia Meléndez, Armando Aguilar-Caballero y Víctor Parra-Tabla, por los comentarios y críticas otorgadas al trabajo. A Melina Ojeda, Alberto Rosado y Joaquín Fernández, por su apoyo en el trabajo de campo y de laboratorio.

\section{Literatura citada}

Andresen, E. 2005. Effects of season and vegetation type on community organization of dung beetles in a tropical dry forest. Biotropica 37:291-300.

Barraza, M. J., F. J. Montes, H. N. Martínez y C. Deloya. 2010. Ensamblaje de escarabajos coprófagos (Scarabaeidae: Scarabaeinae) del bosque tropical seco, Bahía Concha, Santa Marta (Colombia). Revista Colombiana de Entomología 36:285-291.

Bautista, F., E. Batllori, G. Palacio, M. Ortiz y M. Castillo. 2005. Integración del conocimiento actual sobre los paisajes geomorfológicos de la península de Yucatán. In Caracterización y manejo de los suelos de la península de Yucatán. Implicaciones agropecuarias, forestales y ambientales, F. Bautista y A. G. Palacio (eds.). Universidad Autónoma de Campeche/ Universidad Autónoma de Yucatán/ Instituto Nacional de Ecología, México, D.F. p. 33-58.

Bishop, A. L., H. J. McKenzie, L. J. Spohr e I. M. Barchia. 
2005. Interactions between dung beetles (Coleoptera: Scarabaeidae) and the arbovirus vector Culicoides brevitarsis Kieffer (Diptera: Ceratopogonidae). Australian Journal of Entomology 44:89-96.

Breytenbach, W. y J. G. Breytenbach. 1986. Seasonal patterns in dung feeding Scarabaeaidae in the southern Cape. Journal of the Entomological Society of South Africa 49:359-366.

Colwell, R. K. 2009. EstimateS: version 8.20. Statistical estimation of species richness and shared species from samples (Software and user`s guide). http:// www.purl.oclc. org/estimates; última consulta: 20.VII.2009.

Delgado, L., L. N. Peraza y C. Deloya. 2006. Onthophagus yucatanus, a new species of the clypeatus group from Mexico and Guatemala (Coleoptera: Scarabaeidae). Florida Entomologist 89:6-9.

Delgado, L. y B. Kohlmann. 2007. Revisión de las especies del género Uroxys Westwood de México y Guatemala (Coleoptera: Scarabaeidae: Scarabaeinae). Folia Entomológica Mexicana 46:1-36.

Deloya, C. y L. N. Peraza-Flores. 2005. Pedaridium maya (Coleoptera: Scarabaeidae): first record in Yucatán, México. Florida Entomologist 88:341.

Edmonds, D. W. 2003. Tribu Phanaeini. In Atlas de los escarabajos de México. Coleoptera: Lamellicornia, vol. 2, Familias Scarabaeidae, Trogidae, Passalidae y Lucanidae, M. A. Morón (ed.). Argania, Barcelona. p. 58-65.

Escobar, F. 1997. Estudio de la comunidad de coleópteros coprófagos (Scarabaeidae) en un remanente de bosque seco al norte del Tolima, Colombia. Caldasia 19:419-430.

Estrada, A., R. Costa-Estrada, A. A. Dadda y P. Cammarano. 1998. Dung and carrion beetles in tropical rain forest fragments and agricultural habitats at Los Tuxtlas, Mexico. Journal of Tropical Ecology 14:577-593.

Flores, S. e I. Espejel. 1994. Tipos de vegetación de la Península de Yucatán. Serie Etnoflora Yucatanense, fascículo 3, Universidad Autónoma de Yucatán y Universidad de California, Mérida. p. 135.

García-Ramírez, J. C. y L. C. Pardo-Locarno. 2004. Escarabajos Scarabaeinae saprófagos (Coleoptera: Scarabaeidae) en un bosque muy húmedo premontano de los andes occidentales colombianos. Ecología Aplicada 3:59-63

Gill, B. 1991. Dung beetles in tropical American forests. In Dung beetle ecology, I. Hanski e Y. Cambefort (eds.). Princeton University Press, New Jersey. p. 211-229.

Halffter, G. y W. D. Edmonds. 1982. The nesting behavior of dung beetles (Scarabaeinae). An ecological and evolutive approach. Instituto de Ecología/ MAB-UNESCO, México, D.F. p. 176.

Halffter, G. 1991. Historical and ecological factors determining the geographical distribution of beetles (Coleoptera: Scarabaeidae: Scarabaeinae). Folia Entomológica Mexicana 82:195-238
Halffter, G., M. E. Favila y V. Halffter. 1992. A comparative study of the structure of the scarab guild in Mexican tropical rain forest and derived ecosystems. Folia Entomológica Mexicana 84:131-156.

Halffter, G. y L. Arellano. 2002. Response of dung beetle diversity to human-induced changes in a tropical landscape. Biotropica 34:144-154.

Halffter, G. 2003. Tribu Scarabaeini. In Atlas de los escarabajos de México. Coleoptera: Lamellicornia, vol. 2. Familias Scarabaeidae, Trogidae, Passalidae y Lucanidae, M. A. Morón (ed.). Argania, Barcelona. p. 21-43.

Hanski, I. 1989. Dung beetles. In Tropical rain forest ecosystems, H. Lieth y J. Wagner (eds.). Elsevier, Amsterdam. p. 489-511.

Hanski, I. e Y. Cambefort. 1991. Dung Beetle Ecology. Princeton University Press, New Jersey. p. 520.

Horgan, F. G. 2001. Burial of bovine dung by coprophagous beetles (Coleoptera: Scarabaeidae) from horse and cow grazing sites in El Salvador. European Journal of Soil Biology 37:103-111.

Howden, H. y V. Nealis. 1975. Effects of clearing in a tropical rain forest on the composition of the coprophagous scarab beetle fauna (Coleoptera). Biotropica 7:77-85.

Janzen, D. 1983. Seasonal change in abundance of large nocturnal dung beetles (Scarabaedaie) in a Costa Rica deciduous forest and adjacent horse pasture. Oikos 33:274-283.

Klein, B. C. 1989. Effects of forest fragmentation on dung and carrion beetle communities in central Amazonia. Ecology 6:1715-1725.

Kohlmann, B. y A. Solís. 1997. El género Dichotomius (Coleoptera: Scarabaeidae) en Costa Rica. Giornale Italiano di Entomologia 8:343-382.

Kohlmann, B. 2003. The tribe Coprini. In Atlas de los escarabajos de México. Coleoptera: Lamellicornia, vol. 2. Familias Scarabaeidae, Trogidae, Passalidae y Lucanidae, M. A. Morón (ed.). Argania, Barcelona. p. 45-58.

Kohlmann, B. y A. Solís. 2006. El género Canthidium (Coleoptera: Scarabaeidea) en Norteamérica. Giornale Italiano di Entomología 11:235-295.

Larsen, T. y A. Forsyth. 2005. Trap spacing and transect design for dung beetle biodiversity studies. Biotropica 37:322-325.

Martínez, M. I. y E. Montes de Oca. 1994. Observaciones sobre algunos factores microambientales y el ciclo biológico de dos especies de escarabajos rodadores (Coleoptera, Scarabaeidae, Canthon). Folia Entomológica Mexicana 91:47-59.

Montes de Oca, E. y G. Halffter. 1995. Daily and seasonal activities of a guild of the coprophagous, burrowing beetle (Coleoptera: Scarabaeidae: Scarabaeinae) in tropical grasslands. Tropical Zoology 8:159-180.

Montes de Oca, E. 2001. Escarabajos coprófagos de un escenario ganadero típico de la región de los Tuxtlas, Veracruz, México: importancia del paisaje en la composición de un gremio funcional. Acta Zoológica Mexicana (n.s.) 82:111-132. 
Morón, M. A. 2003. Atlas de los escarabajos de México. Coleoptera: Lamellicornia, vol. 2. Familias Scarabaeidae, Trogidae, Passalidae y Lucanidae. Argania, Barcelona. p. 227.

Navarro-Reyes, M. G. 2005. Plan Municipal de Desarrollo Rural Sustentable, Tizimín, Yucatán. Secretaría de Agricultura, Ganadería, Desarrollo Rural, Pesca y Alimentación. p. 28.

Nichols, E., S. Spector, J. Louzada, T. Larsen, S. Amezquita y M. Favila. 2008. Ecological functions and ecosystem services provided by Scarabaeinae dung beetles. Biological Conservation 141:1461-1474.

Peraza, L. N. y C. Deloya. 2006. A new mexican species of Dichotomius hope (Coleoptera: Scarabaeidae) and a key to species of the Carolinus group. Neotropical Entomology 35:629-631.

Reyes-Novelo, E., H. Delfín-González y M. A. Morón. 2007. Copro-necrophagous beetle (Coleoptera, Scarabaeidae, Scarabaeinae) diversity in an agroecosystem in Yucatan, Mexico. Revista de Biología Tropical 55:83-99.

Rivera-Cervantes, L. E. y G. Halffter. 1999. Monografía de las especies mexicanas de Canthon del subgénero Glaphyrocanthon (Coleoptera: Scarabaeidae: Scarabaeinae). Acta Zoológica Mexicana 77:23-150.

Rougon, D. y C. Rougon. 1991. Dung beetles of the Sahel region. In Dung beetle ecology, I. Hanski e Y. Cambefort (eds.). Princeton University Press, New Jersey. p. 230-254.

Steinfeld, H., P. Gerber, T. Wassenaar, V. Castel, M. Rosales y C. Haan. 2006. Livestock's long shadow: environmental issues and options, Food and Agriculture Organization of the United Nations, Rome. p. 414.

Vaz-de-Mello, F. Z., W. D. Edmonds, F. C. Ocampo y P. Schoolmeesters. 2011. A multilingual key to the genera and subgenera of the subfamily Scarabaeinae of the New World (Coleoptera: Scarabaeidae). Zootaxa 2854:1-73

Wolda, H. 1988. Insect seasonality. Annual Review of Ecology and Systematics 19:1-18.

Zunino, M. 2003. Tribu Onthophagini. In Atlas de los escarabajos de México. Coleoptera: Lamellicornia, Volumen 2. Familias Scarabaeidae, Trogidae, Passalidae y Lucanidae, M. A. Morón (ed.). Argania, Barcelona. p. 66-74. 